\title{
A New Depth Classification Method based on Underwater Acoustics for Naval Defense Applications
}

\author{
Orhan Yaman ${ }^{1}$, Emrah Aydemir ${ }^{2 *}$ \\ ${ }^{1}$ Firat University, Faculty of Technology, Department of Digital Forensics Engineering, Elazig, Turkey, (ORCID: 0000-0001-9623-2284), orhanyaman@ firat.edu.tr \\ ${ }^{2 *}$ Sakarya University, Faculty of Business, Department of Management Information Systems, Sakarya, Turkey, (ORCID: 0000-0002-8380-7891), \\ emrahaydemir@sakarya.edu.tr
}

(First received 27 September 2021 and in final form 3 November 2021)

(DOI: 10.31590/ejosat.1001051)

ATIF/REFERENCE: Yaman, O. \& Aydemir, E. (2021). A New Depth Classification Method based on Underwater Acoustics for Naval Defense Applications. European Journal of Science and Technology, (31), 1-7.

\begin{abstract}
The main purpose of this research is to present an automatic underwater acoustic classification model with high performance. Thus, a new sound dataset was collected. By using this dataset, a new underwater depth classification method is proposed in this work. Average pooling has been used to pre-processing underwater sounds. The used average pooling model is both removed the noises and compressed signal. S-transform and AlexNet have been used for feature extraction. By deploying S-transform to underwater sounds, contour images have been obtained. These images have been utilized input of the AlexNet. Herein, AlexNet has been utilized to extract features by using transfer learning. Features extracted have been classified with the Support Vector Machine (SVM). In our method, 99.05\% accuracy has been calculated. The calculated results and findings illustrate the success of our proposed S-transform and AlexNet based model on the underwater sound classification.
\end{abstract}

\section{Deniz Savunma Uygulamaları için Sualtı Akustiğine Dayalı Yeni Bir Derinlik Sinıflandırma Yöntemi}

$\ddot{O} \mathbf{z}$

$\mathrm{Bu}$ araştırmanın temel amacı, yüksek performanslı otomatik bir sualtı akustik sınıflandırma modeli sunmaktır. Böylece yeni bir ses veri seti toplanmıştır. Bu veri seti kullanılarak, bu çalışmada yeni bir sualtı derinlik sınıflandırma yöntemi önerilmiştir. Sualtı seslerinin ön işlemesi için ortalama havuzlama kullanılmıştır. Kullanılan ortalama havuzlama modeli hem gürültüleri hem de sıkıştırılmış sinyali ortadan kaldırmıştır. Özellik çıkarımı için S-dönüşüm ve AlexNet kullanılmıştır. S-dönüşümünün su altı seslerine yerleştirilmesiyle kontur görüntüleri elde edilmiştir. Bu görüntüler AlexNet'in girdisi olarak kullanılmıştır. Burada, transfer öğrenme kullanılarak öznitelikleri çıkarmak için AlexNet kullanılmıştır. Çıkarılan özellikler Destek Vektör Makinesi (SVM) ile sınıflandırılmıştır. Bizim yöntemimizde \%99,05 doğruluk hesaplanmıştır. Hesaplanan sonuçlar ve bulgular, su altı ses sınıflandırmasında önerilen S-dönüşüm ve AlexNet tabanlı modelimizin başarısını açıkça göstermektedir.

Anahtar Kelimeler: Sualtı ses sınıflandırması; S-dönüşümü; Derin Öğrenme; AlexNet; SVM.

\footnotetext{
* Corresponding Author: emrahaydemir@ sakarya.edu.tr
} 


\section{Introduction}

Underwater systems are important for naval defense applications. Sound-based applications have been developed for underwater monitoring and object detection. Underwater sounds can be used for both civil and military applications. Submarine vehicles can be detected by using underwater sounds in military applications. Moreover, applications are being developed for depth and direction detection with underwater sounds. In the literature, object detection is made using underwater radar and images (Fan et al., 2018; Neves et al., 2020; Song et al., 2018). However, there are few studies for underwater sound systems.

Firat et al. (Firat et al., 2017), detected ship noise caused by propellers and machinery. They collected underwater sounds using different marine vehicles such as cruise boats, cargo ships, and passenger boats. They applied Demon and Lofar methods to underwater sounds. Liu et al. method (Liu et al., 2021), used the Convolutional Neural Network (CNN) and determined the direction of the target underwater. According to Jiang et al. (Jiang, Wu, et al., 2020), proposed a sound-based method for underwater target recognition. Sound signals obtained underwater are noisy. They used signal preprocessing to reduce these noises. They obtained the spectrogram of the preprocessed sound signals. The results were computed using CNN and machine learning using spectrograms.

Dong et al. (Dong et al., 2021) provide an efficient method for the recognition of unstable underwater acoustic datasets. They proposed a convolutional neural network and an entropybased method. The proposed approach was applied on unstable underwater acoustic datasets of targets and communication signals. Time-frequency conversions of unbalanced acoustic signals were obtained. Time-frequency transformations were converted to images and given as an input to the CNN model. The used proposed method, $90 \%$ accuracy was calculated. Jiang et al. (Jiang, Shi, et al., 2020) proposed a multi-scale spectral method for noise removal and target recognition in underwater acoustic signals. In this study, the noises in the Acoustic data set were removed and the target was determined by machine learning. They calculated about $90 \%$ accuracy in the proposed method. Hu et al. (Hu et al., 2018) proposed an extreme learning machine (ELM) and CNN-based method for underwater noise removal. Feature extraction was performed from underwater acoustic signals using the CNN model. Extracted features were classified using ELM. They calculated a 93.04\% recognition rate in the experiments performed on the dataset of civilian ships. Wang et al. (Wang et al., 2019) developed multidimensional fusion features and modified deep neural network (MFFMDNN) based method to recognize underwater acoustic targets. Underwater acoustic feature extraction was performed using gammatone frequency cepstral coefficient (GFCC) and modified empirical mode decomposition (MEMD) methods. The proposed method calculated $94.3 \%$ accuracy with 800 iterations. Yaman et al. (Yaman, Tuncer, et al., 2021) identified propeller types using underwater acoustic sounds. They collected an underwater acoustic dataset. They developed a DES pattern algorithm to extract features from these sound signals. Decision Tree, $\mathrm{k}$ Nearest Neighbor (KNN), and Support Vector Machine (SVM) algorithms were used to classify the obtained features. They calculated $99.8 \%$ accuracy using the SVM algorithm.

Our motivation in this study is to collect a new dataset and propose a new hybrid method for underwater depth detection.
Sounds have been collected at depths of $5,10,15,20,25$, and 30 meters underwater. This sound data has been recorded with 44,100 kHz. Average pooling has been applied in the signalpreprocessing step. The data collected 44100 times in 1 second has been reduced to 441 sizes. In the literature, $44,100 \mathrm{kHz}$ sound signals are divided into 1-second samples for feature extraction (Altinors et al., 2021). For this reason, underwater sound signals collected in this study have been used by dividing into 1-second samples. The feature has been extracted with $1 \times 441$ signal S-transform and these features have been recorded as figures. S-transform images have been used with AlexNet and $1 \times 1000$ features have been extracted. Extracted features have been classified by SVM. In the proposed method, a hybrid model is developed using both S-transform + AlexNet and SVM. In this study, a contribution to the literature has been made with the proposed method and underwater dataset.

\section{Materials}

In this study, an underwater depth dataset has been collected using a sound/video recorder. Features of the used sound/video recorder; QuickTime Video File (MOV), the bit rate is 1411 kbps, channels 2 (stereo), the Sample rate is $44.100 \mathrm{kHz}$. We collected the underwater sound dataset near the Keban dam / Turkey. The experimental setup developed to collect the underwater depth dataset can be seen in Figure 1.

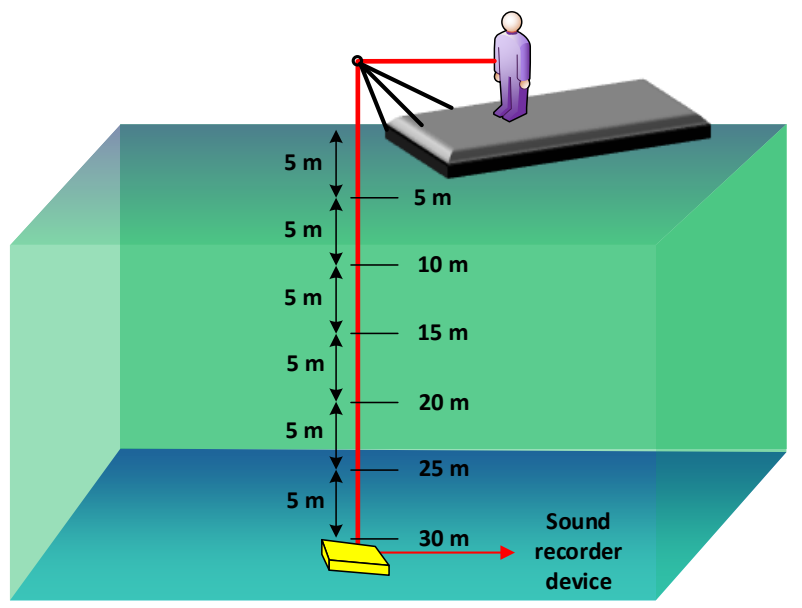

Fig. 1. Experimental setup developed for the collection of underwater depth dataset

As can be seen in Figure 1, the sound recorder has been used between five and thirty meters. The sound recorder has been fixed under the water at six points. Five minutes of sounds have been collected for each point. The features of the underwater depth dataset collected are shown in Table 1.

Table 1. Class information of the collected underwater direction dataset

\begin{tabular}{l|l|l|l}
\hline Class number & Class definition & $\begin{array}{l}\text { Time } \\
(\mathbf{m i n})\end{array}$ & $\begin{array}{l}\text { Number of } \\
\text { Samples }\end{array}$ \\
\hline Class 1 & Five meters & 5 & 300 \\
\hline Class 2 & Ten meters & 5 & 300 \\
\hline Class 3 & Fifteen meters & 5 & 300 \\
\hline Class 4 & Twenty meters & 5 & 300 \\
\hline Class 5 & Twenty-five meters & 5 & 300 \\
\hline Class 6 & Thirty meters & 5 & 300 \\
\hline
\end{tabular}


In the depth dataset, we obtained $300 \times 44100$ data by taking 300 seconds of sound for each class. In this case, $1800 \times 44100$ data has been collected for six classes in the depth dataset. In this study, sample sound signals from microphones for the depth dataset are drawn in Figure 2.

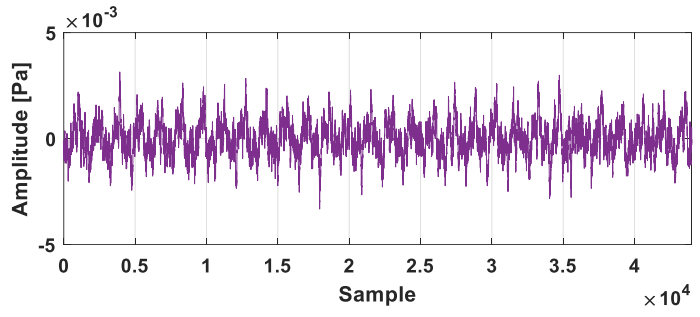

a)

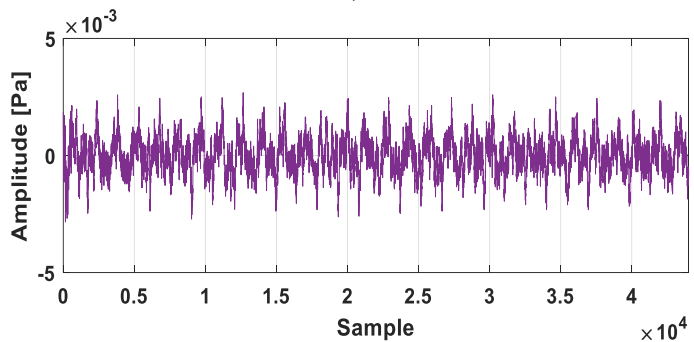

b)

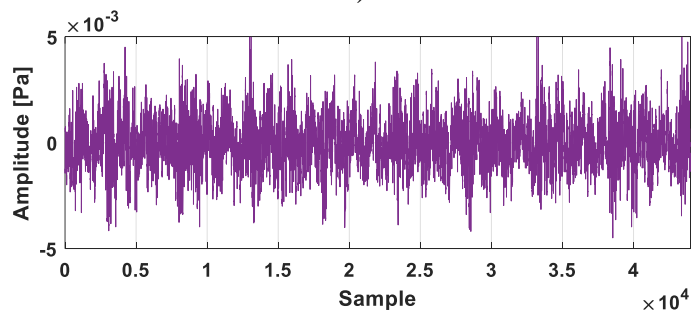

c)

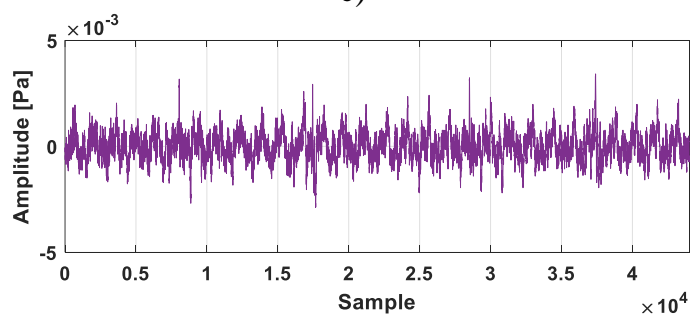

d)

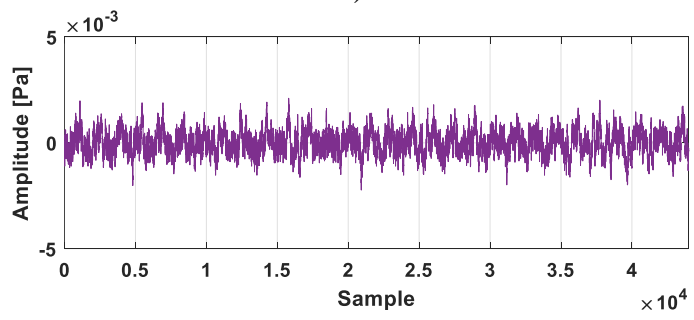

e)

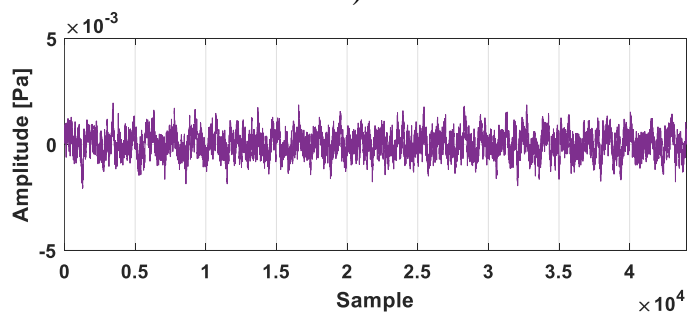

f)

Fig. 2. Sound samples collected for direction determination a) Class 1 b) Class 2 c) Class 3 d) Class 4 e) Class 5 f) Class 6

\section{Proposed Method}

In this study, a new method for underwater depth classification is proposed. The block diagram of the proposed method is summarized in Figure 3.

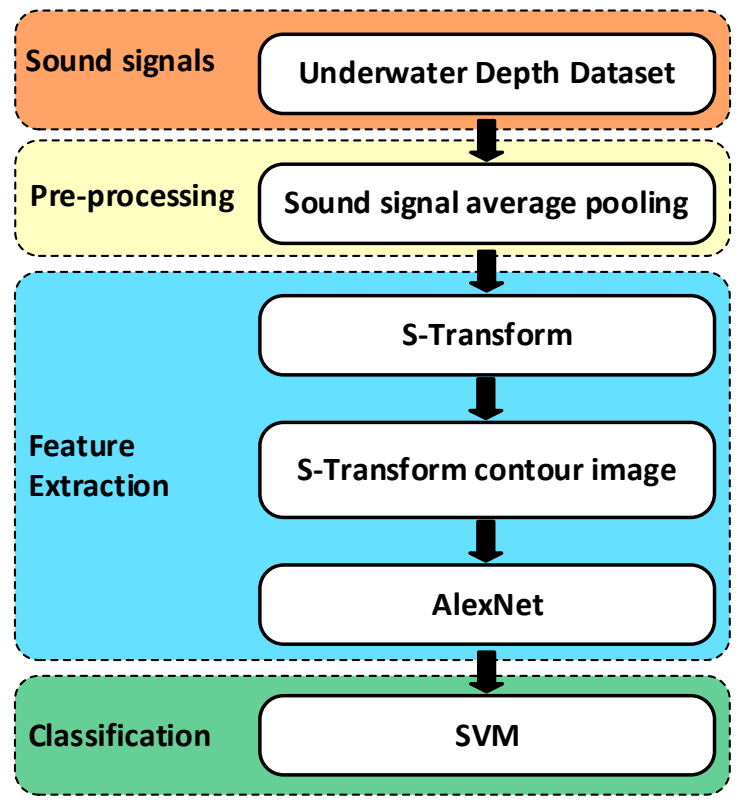

Fig. 3. Block diagram of the proposed underwater depth classification method

In the first step of the proposed method, we have preprocessed the underwater depth dataset. In the preprocessing step, average pooling has been applied to the signal. $1 \times 44100$ samples have been collected in 1 second. This example transforms into $1 \times 441$ after the average pooling step. The average pooling result of the sample signal drawn in Figure 2.a) can be seen in Figure 4.

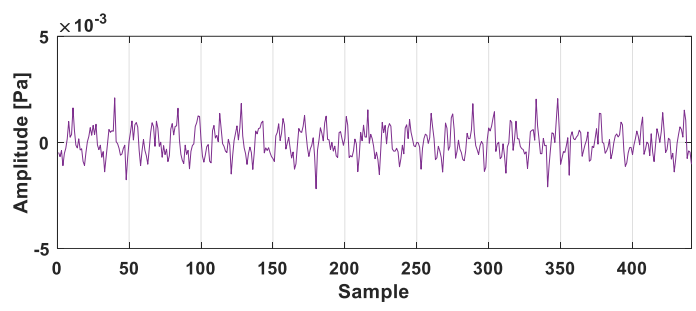

Fig. 4. Average pooling result of a sample signal (Fig.2.a)

By using averaging pooling, noise in the signal is reduced. This process has been repeated for a total of 1800 samples, which have six classes. $1800 \times 44100$ data is reduced to $1800 \times 441$ size. Because the size of the sound signals is decreased, the feature extraction time is lowered. Short-term Fourier and Gabor transform is used in fixed-width windows (Das et al., 2013; Gowtham et al., 2019). These transformation methods are insufficient in the analysis of non-stationary signals. In addition, wavelet transforms do not achieve high success in noisy signals. S-transform has been developed to solve these problems (Stockwell, 2007). The S-transform consists of a shorttime Fourier transform and a continuous wavelet transform. Unlike wavelet transform, S-transform gives frequencydependent time-frequency information. The obtained timefrequency information represents the instantaneous phase information. This phase information corresponds to the phase 
obtained by the Fourier transform. The S-transform is given in equation 1 (Stockwell, 1996).

$S T(\tau, f)=\int_{-\infty}^{\infty} \beta(t) \frac{|f|}{\sqrt{2 \pi}} e^{\left[-\frac{f^{2}(\tau-t)^{2}}{2}\right]} e^{-i 2 \pi f t} d t$

In Equation 1, the parameter $\tau$ represents the time axis, and the parameter $f$ represents the frequency axis. The parameter $\beta(t)$ represents the input function, and $\frac{|f|}{\sqrt{2 \pi}} e^{\left[-\frac{f^{2}(\tau-t)^{2}}{2}\right]}$ represents the Gaussian window. In Figure 2, average pooling and S-transform have been applied to the sample signals. The images obtained after $\mathrm{S}$-transform can be seen in Figure 5.

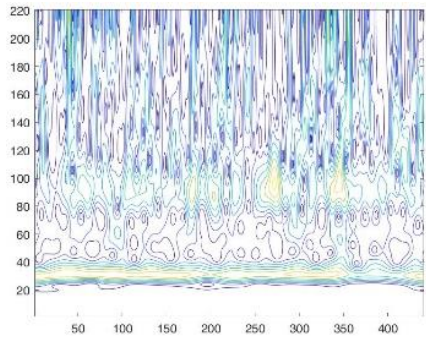

a)

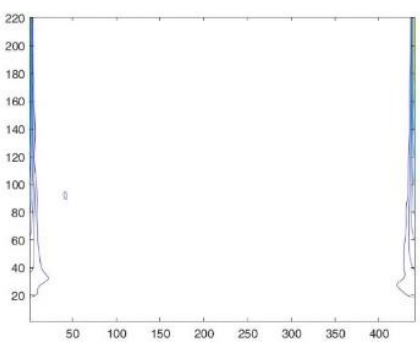

c)

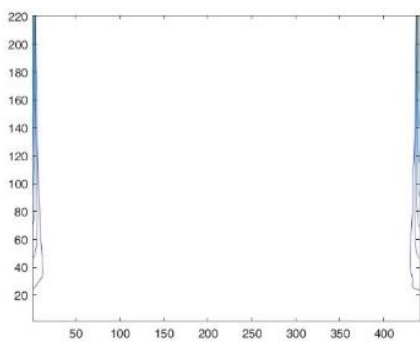

e)

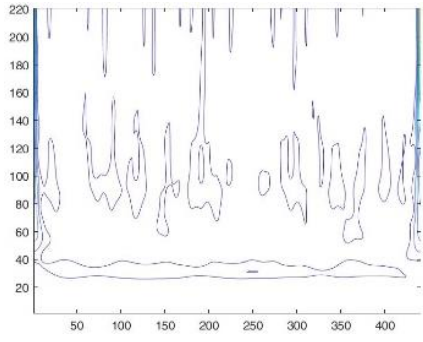

b)

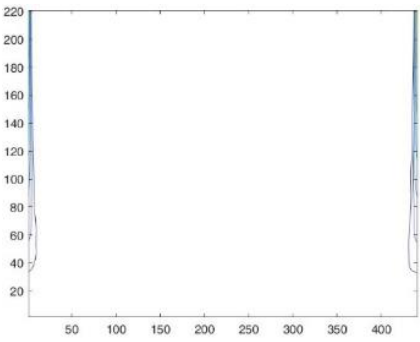

d)

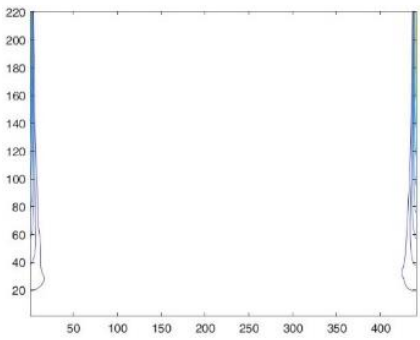

f)
Fig. 5. S-transform conversion of underwater depth sounds a) Class 1 b) Class 2 c) Class 3 d) Class 4 e) Class 5 f) Class 6 (see Fig.2)

As can be seen in Figure 5, S-transform contour images have been obtained from the sample signals. This process has been repeated for six classes. As a result of the S-transform, 1800 images have been obtained. Feature extraction has been performed from these images with pre-trained deep learning models. The operation time and accuracy of the models have been examined to select the best performing deep learning model. Feature extraction has been performed using S-transform images with AlexNet, DarkNet19, DarkNet53, DenseNet201, GoogleNet, InceptionResNetV2, InceptionV3, MobileNetV2,
NasNetLarge, NasNetMobile, ResNet18, ResNet50, ResNet101, ShuffleNet, Vgg16, Vgg19 and XceptionNet deep models. These models are pre-trained models and transfer learning has been applied by using these deep networks. The pre-trained networks were trained on the ImageNet dataset. The performance results calculated for these pre-trained deep learning models can be seen in Figure 6.

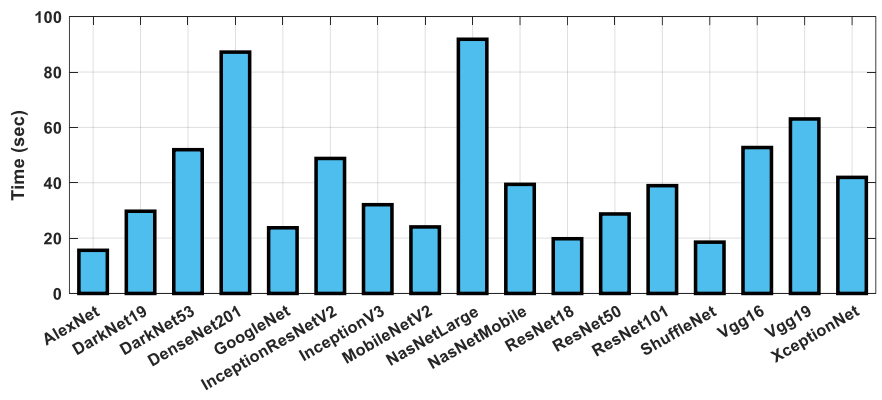

a)

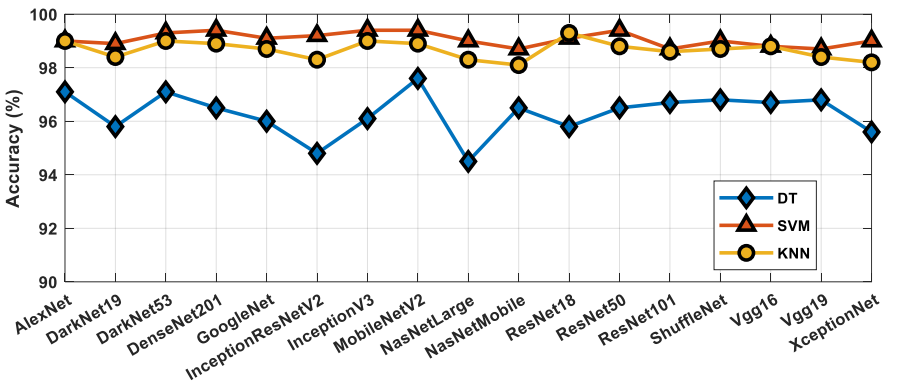

b)

Fig.6. Performance results of pre-trained deep learning models a) Operation time of models b) Classification results of models

In Figure 6.a), the feature extraction times of the pre-trained deep learning models have been computed. The feature extraction time from 1800 images ranges from 15.59 seconds to 91.85 seconds. The fastest feature extraction for the underwater depth dataset used in this study has been calculated with AlexNet. Thus, we have used AlexNet as a feature extractor. In addition, extracted features are classified by Decision Tree(DT)(Bedi et al., 2021), Support Vector Machine (SVM)(Bayğın et al., 2021), and K-Nearest Neighbors (KNN)(Aydemir et al., 2020) algorithms. AlexNet is preferred for feature extraction because of its performance in both operation time and accuracy (Haryanto et al., 2020).

AlexNet consists of eight layers (Krizhevsky et al., 2012) and architecture has significantly increased the ImageNet classification accuracy. AlexNet contains five convolutional layers and three fully connected layers.

In this study, the pre-trained AlexNet model has been used and 1800x1000 features have been extracted for 1800 images. Obtained features have been classified by the Cubic SVM algorithm. Before choosing the Cubic SVM algorithm, the performances of twelve classifiers were tested. These used classifiers in tests; are Fine DT, Medium DT, Coarse DT, Linear Discriminant, Gaussian Naïve Bayes, Linear SVM, Quadratic SVM, Cubic SVM, Fine KNN, Medium KNN, Cosine KNN, and Ensemble Boosted Trees. The calculated accuracies for these classifiers have been demonstrated in Figure 7. 


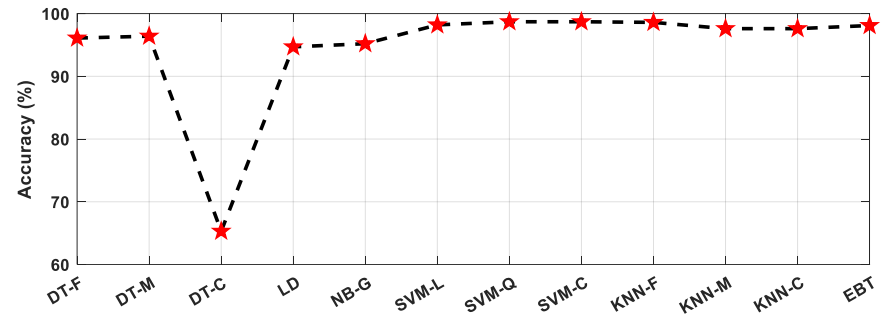

Fig. 7. The accuracy results computed for Fine DT (DT-F), Medium DT (DT-M), Coarse DT (DT-C), Linear Discriminant (LD), Gaussian Nä̈ve Bayes (NB-G), Linear SVM (SVM-L), Quadratic SVM (SVM-Q), Cubic SVM (SVM-C), Fine KNN $(K N N-F)$, Medium KNN (KNN-M), Cosine KNN (KNN-C), and Ensemble Boosted Trees (EBT)

As can be seen in Figure 7, the highest accuracy has been calculated with Cubic SVM. Therefore, extracted features are classified with Cubic SVM. The parameters of the Cubic SVM algorithm used in the proposed method are tabulated in Table 2.

Table 2. Parameters of SVM algorithms used in the proposed method

\begin{tabular}{l|l}
\hline Parameters & Cubic SVM \\
\hline Kernel function & Cubic \\
\hline Box constraint level & 1 \\
\hline Kernel scale mode & Auto \\
\hline Kernel scale & 1 \\
\hline Multiclass method & One-vs-one \\
\hline
\end{tabular}

\section{Experimental Results}

In this study, the proposed method has been applied on a computer with i7-9700 CPU 3.00 GHz, 32GB RAM, and 64-bit Windows 10 operating system. In the proposed method, preprocessing and feature extraction steps are developed in the MATLAB 2020a program with $m$-file. The classification process has been calculated using the MATLAB Classification Learner Toolbox. The confusion matrix has been computed using 10-fold cross-validation. Confusion matrix calculated with 10 -fold crossvalidation can be seen in Figure 8.

\begin{tabular}{|c|c|c|c|c|c|c|}
\multicolumn{7}{c|}{ Predicted Class } \\
\cline { 2 - 8 } \multicolumn{1}{c|}{} & 1 & 2 & 3 & 4 & 5 & 6 \\
\hline 1 & 298 & 1 & 0 & 0 & 0 & 1 \\
\hline 2 & 0 & 299 & 1 & 0 & 0 & 0 \\
\hline 3 & 0 & 0 & 296 & 0 & 2 & 2 \\
\hline 4 & 0 & 0 & 1 & 299 & 0 & 0 \\
\hline 5 & 0 & 0 & 2 & 0 & 298 & 0 \\
\hline 6 & 0 & 0 & 7 & 0 & 0 & 293 \\
\hline
\end{tabular}

\section{Fig. 8. Confusion Matrix obtained for Cubic SVM}

In the proposed method, 1000 iterations have been run to get results with the Cubic SVM classification method. Accuracy, Precision, Recall, Geometric mean, and F1-score results computed for Cubic SVM are presented in Table 3.
Table 3. Accuracy, precision, recall, geometric mean, and F1score (\%) results of the used underwater depth acoustic classifiers.

\begin{tabular}{|l|l|l|l|l|l|l|}
\hline Classifiers & Statistics & Accuracy & Precision & Recall & $\begin{array}{l}\text { Geometric } \\
\text { mean }\end{array}$ & $\begin{array}{l}\text { F1- } \\
\text { score }\end{array}$ \\
\hline \multirow{4}{*}{$\begin{array}{l}\text { Cubic } \\
\text { SVM }\end{array}$} & Maximum & 99.05 & 99.06 & 99.05 & 99.05 & 99.06 \\
\cline { 2 - 7 } & Minimum & 98.16 & 98.67 & 98.66 & 98.65 & 98.67 \\
\cline { 2 - 7 } & Mean & 98.63 & 99.01 & 98.99 & 98.99 & 99.0 \\
\cline { 2 - 7 } & $\begin{array}{l}\text { Standard } \\
\text { deviation }\end{array}$ & 0.12 & 0.07 & 0.07 & 0.07 & 0.07 \\
\hline
\end{tabular}

Maximum accuracy, precision, recall, geometric mean, and F1-Score have been calculated as $99.05 \%, 99.06 \%, 99.05 \%$, $99.05 \%$, and $99.06 \%$, respectively, with 1000 iterations of the Cubic SVM algorithm. In this study, 10-fold cross-validation has been used to calculate the classification results. In the proposed method, Fold-wise results for Cubic SVM have been computed and shown in Figure 9.

\begin{tabular}{|c|c|c|c|c|c|c|c|c|c|c|c|}
\hline \multirow[b]{2}{*}{ Fold-1 } & & $\% 10$ & \multicolumn{4}{|c|}{ Training Data } & $\overline{\% 10}$ & \multicolumn{3}{|c|}{ Testing Data } & $\begin{array}{l}\text { Accuracy } \\
\text { (\%) }\end{array}$ \\
\hline & $\% 10$ & $\% 10$ & $\% 10$ & $\% 10$ & $\% 10$ & $\% 10$ & $\% 10$ & $\% 10$ & $\% 10$ & $\% 10$ & 98.89 \\
\hline Id -2 & $\% 10$ & $\% 10$ & $\% 10$ & $\% 10$ & $\% 10$ & $\% 10$ & $\% 10$ & $\% 10$ & $\% 10$ & $\% 10$ & \\
\hline Id-3 -3 & $\% 10$ & $\% 10$ & $\% 10$ & $\% 10$ & $\% 10$ & $\% 10$ & $\% 10$ & $\% 10$ & $\% 10$ & $\% 10$ & \\
\hline Id-4 & $\% 10$ & $\% 10$ & $\% 10$ & $\% 10$ & $\% 10$ & $\% 10$ & $\% 10$ & $\% 10$ & $\% 10$ & $\% 10$ & 98.33 \\
\hline old-5 & $\% 10$ & $\% 10$ & $\% 10$ & $\% 10$ & $\% 10$ & $\% 10$ & $\% 10$ & $\% 10$ & $\% 10$ & $\% 10$ & 98.89 \\
\hline old-6 & $\% 10$ & $\% 10$ & $\% 10$ & $\% 10$ & $\% 10$ & $\% 10$ & $\% 10$ & $\% 10$ & $\% 10$ & $\% 10$ & 100 \\
\hline Fold-7 & $\% 10$ & $\% 10$ & $\% 10$ & $\% 10$ & $\% 10$ & $\% 10$ & $\% 10$ & $\% 10$ & $\% 10$ & $\% 10$ & 97.22 \\
\hline Fold-8 & $\% 10$ & $\% 10$ & $\% 10$ & $\% 10$ & $\% 10$ & $\% 10$ & $\% 10$ & $\% 10$ & $\% 10$ & $\% 10$ & 99.44 \\
\hline old-9 & $\% 10$ & $\% 10$ & $\% 10$ & $\% 10$ & $\% 10$ & $\% 10$ & $\% 10$ & $\% 10$ & $\% 10$ & $\% 10$ & 100 \\
\hline Id -10 & $\% 10$ & $\% 10$ & $\% 10$ & $\% 10$ & $\% 10$ & \%10 & $\% 10$ & $\% 10$ & $\% 10$ & $\% 10$ & 98.33 \\
\hline
\end{tabular}

Fig. 9. The plot of best accuracy (\%) obtained for different folds using underwater depth dataset

As seen in Figure 9, the results of Fold-1, Fold-2, Fold-3... Fold-10 have been calculated for the Cubic SVM classification algorithm. The highest results have been seen in Fold-2, Fold-6, Fold-9 and the lowest result have been seen in Fold-7. Our method Class by Class results are shown in Figure 10.
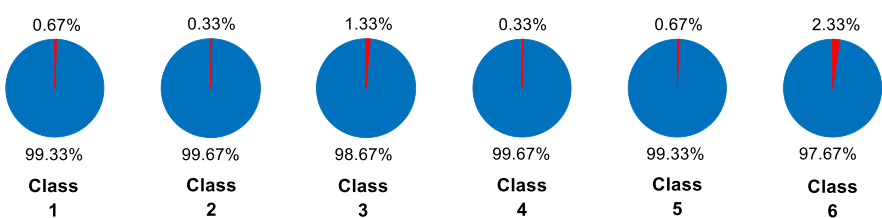

Fig. 10. Classification accuracies (\%) obtained for various classes using our proposed method with an underwater depth dataset

As seen in Figure 10, the best results have been obtained in Class 2 and Class 4, while the lowest results have been illustrated in Class 6. Class by Class results support the accuracy of the confusion matrix obtained in the study.

\section{Conclusion and Discussions}

Underwater acoustic systems are widely used in both civil and military applications. Vehicles such as ships, boats, and submarines can be detected using underwater sounds. In addition, the underwater depths of these vehicles can be known. In this study, depth classification has been made using 
underwater sounds. A microphone has been used underwater and sounds have been collected for five minutes from six different depths. Preprocessing has been done with average pooling in the underwater depth dataset. S-transform and AlexNet have been used for feature extraction. The Cubic SVM algorithm has classified extracted features. $99.05 \%$ accuracy has been calculated with the proposed method for six classes. There are very few studies on underwater sound classification in the literature. The related state-of-art studies to our model are listed in Table 4.

Table 4. Comparison of the results of the proposed method with the literature

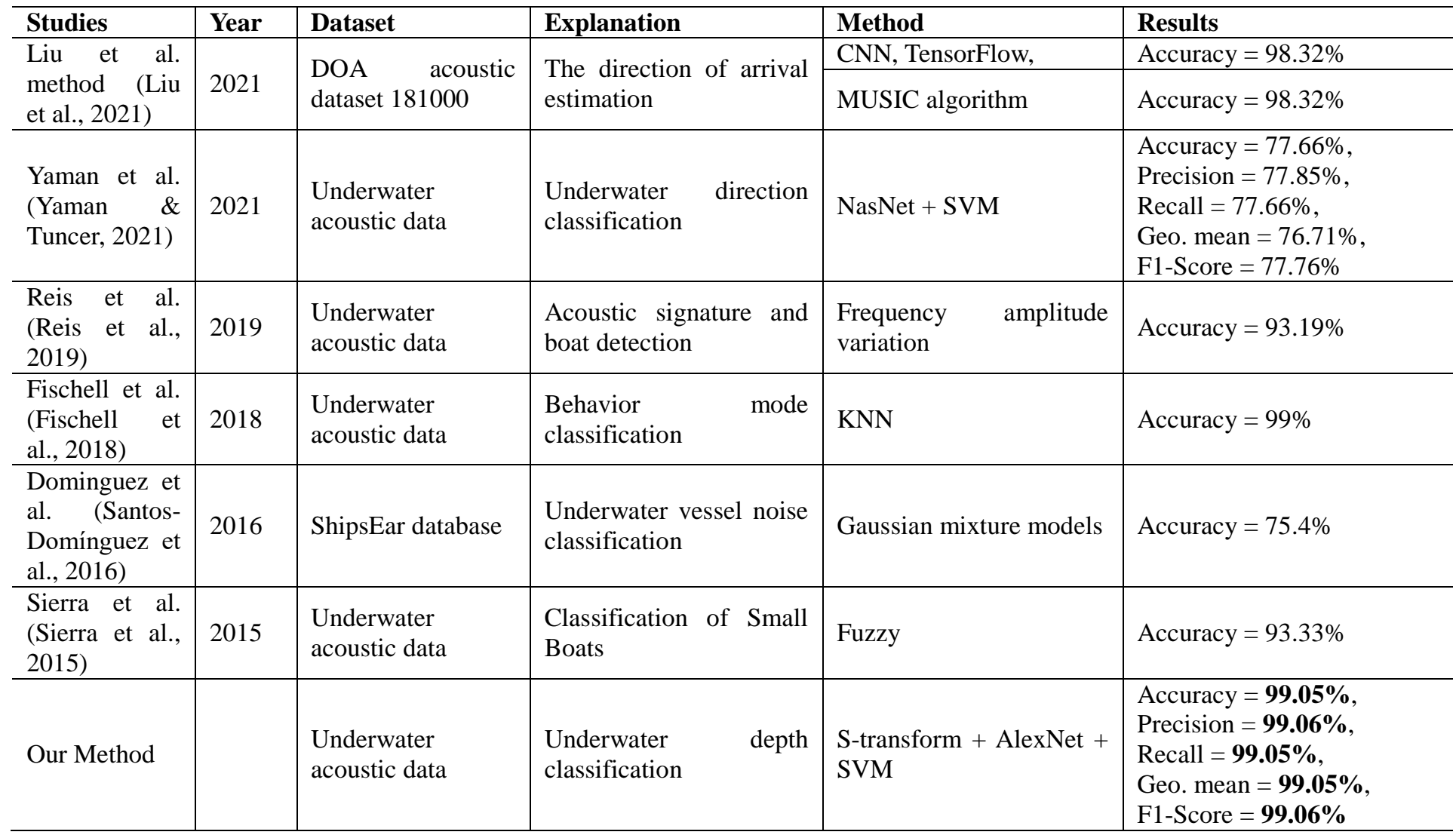

The proposed method has been compared with the literature, and the proposed method has higher accuracy. According to Yaman et al. (Yaman \& Tuncer, 2021) determined the direction of vehicles moving underwater with underwater sound data in their study. They used NasNet deep learning model and SVM in their study, and $77.66 \%$ accuracy was calculated. Fischell et al. (Fischell et al., 2018) proposed a KNN-based method to determine the underwater position of underwater vehicles. Their method calculated $99 \%$ accuracy. On the other hand, Sierra et al. (Sierra et al., 2015) proposed a fuzzy-based method for detecting speedboats, and $93.33 \%$ accuracy was calculated.

\section{Future Works}

Studies are carried out in both civil and military fields by using underwater acoustic signals. Due to underwater acoustic signals, there are many studies such as underwater target detection, detection of leaks in pipelines, and object detection. In this study, depth detection has been performed using underwater sound signals. In future studies, it is aimed to collect acoustic datasets in deeper regions. In addition, underwater target detection methods will be developed with the developed methods. Methods will be developed to detect the location of the target by collecting underwater sound signals from many areas.

\section{Acknowledgment}

This work is supported by Firat University Research Fund, Turkey Project Number: MMY.20.01

\section{References}

Altinors, A., Yol, F., \& Yaman, O. (2021). A sound based method for fault detection with statistical feature extraction in UAV motors. Applied Acoustics, 183, 108325. doi: 10.1016/j.apacoust.2021.108325

Aydemir, E., Tuncer, T., \& Dogan, S. (2020). A Tunable-Q wavelet transform and quadruple symmetric pattern based EEG signal classification method. Medical Hypotheses, 134(December 2019), $109519 . \quad$ doi: 10.1016/j.mehy.2019.109519

Bayğın, M., Yaman, O., \& Tuncer, T. (2021). Akıllı Şehirler için Özellik Çıkarımı ve Makine Öğrenmesi Tabanlı Asfalt Durum İzleme Yaklaşımı. European Journal of Science and Technology, 23, 81-88. doi: 10.31590/ejosat.844592

Bedi, P., Mewada, S., Vatti, R. A., Singh, C., Dhindsa, K. S., Ponnusamy, M., \& Sikarwar, R. (2021). Detection of attacks in IoT sensors networks using machine learning algorithm. 
Microprocessors and Microsystems, 82(December 2020), 103814. doi: 10.1016/j.micpro.2020.103814

Das, M. K., \& Ari, S. (2013). Analysis of ECG signal denoising method based on S-transform. Irbm, 34(6), 362-370. doi: 10.1016/j.irbm.2013.07.012

Dong, Y., Shen, X., Jiang, Z., \& Wang, H. (2021). Recognition of imbalanced underwater acoustic datasets with exponentially weighted cross-entropy loss. Applied Acoustics, $\quad 174, \quad 107740 . \quad$ doi: 10.1016/j.apacoust.2020.107740

Fan, X., Wu, J., Shi, P., Zhang, X., \& Xie, Y. (2018). A novel automatic dam crack detection algorithm based on localglobal clustering. Multimedia Tools and Applications, 77(20), 26581-26599. doi: 10.1007/s11042-018-5880-1

Fischell, E. M., Viquez, O., \& Schmidt, H. (2018). Passive acoustic tracking for behavior mode classification between surface and underwater vehicles. IEEE International Conference on Intelligent Robots and Systems, 2383-2388. doi: 10.1109/IROS.2018.8593981

Fırat, U., \& Akgül, T. (2017). Gemi Akustik İz Analizi. EMO Bilimsel Dergi, 7(13), 25-31.

Gowtham, S., Keerthana, I., \& Balaji, M. (2019). Characterization and Classification of Hall Sensor Faults using S-Transform Analysis on BLDC Motor Drive. 2019 IEEE 1st International Conference on Energy, Systems and Information Processing, ICESIP 2019. doi: 10.1109/ICESIP46348.2019.8938284

Haryanto, T., Sitanggang, I. S., Agmalaro, M. A., \& Rulaningtyas, R. (2020). The Utilization of Padding Scheme on Convolutional Neural Network for Cervical Cell Images Classification. CENIM 2020 - Proceeding: International Conference on Computer Engineering, Network, and Intelligent Multimedia 2020, 34-38. doi: 10.1109/CENIM51130.2020.9297895

Hu, G., Wang, K., Peng, Y., Qiu, M., Shi, J., \& Liu, L. (2018). Deep Learning Methods for Underwater Target Feature Extraction and Recognition. Computational Intelligence and Neuroscience, 2018. doi: 10.1155/2018/1214301

Jiang, J., Shi, T., Huang, M., \& Xiao, Z. (2020). Multi-scale spectral feature extraction for underwater acoustic target recognition. Measurement: Journal of the International Measurement Confederation, 166, 108227. doi: 10.1016/j.measurement.2020.108227

Jiang, J., Wu, Z., Lu, J., Huang, M., \& Xiao, Z. (2020). Interpretable features for underwater acoustic target recognition. Measurement: Journal of the International Measurement Confederation, $108586 . \quad$ doi: 10.1016/j.measurement.2020.108586

Krizhevsky, A., Sutskever, I., \& Hinton, G. E. (2012). ImageNet Classification with Deep Convolutional Neural Networks. Advances in Neural Information Processing Systems, 25, 1097-1105. doi: 10.1201/9781420010749

Liu, Y., Chen, H., \& Wang, B. (2021). DOA estimation based on CNN for underwater acoustic array. Applied Acoustics, 172, 107594. doi: 10.1016/j.apacoust.2020.107594

Neves, G., Ruiz, M., Fontinele, J., \& Oliveira, L. (2020).
Rotated object detection with forward-looking sonar in underwater applications. Expert Systems with Applications, 140, 112870. doi: 10.1016/j.eswa.2019.112870

Reis, C. D. G., Padovese, L. R., \& de Oliveira, M. C. F. (2019). Automatic detection of vessel signatures in audio recordings with spectral amplitude variation signature. Methods in Ecology and Evolution, 10(9), 1501-1516. doi: 10.1111/2041-210X.13245

Santos-Domínguez, D., Torres-Guijarro, S., Cardenal-López, A., \& Pena-Gimenez, A. (2016). ShipsEar: An underwater vessel noise database. Applied Acoustics, 113, 64-69. doi: 10.1016/j.apacoust.2016.06.008

Sierra, E., \& Contreras, J. (2015). Classification of small boats using fuzzy classifier. Annual Conference of the North American Fuzzy Information Processing Society - NAFIPS, 2015-Septe, 0-4. doi: 10.1109/NAFIPSWConSC.2015.7284174

Song, W., Wang, Y., Huang, D., \& Tjondronegoro, D. (2018). A rapid scene depth estimation model based on underwater light attenuation prior for underwater image restoration. Lecture Notes in Computer Science (Including Subseries Lecture Notes in Artificial Intelligence and Lecture Notes in Bioinformatics), 11164 LNCS, 678-688. doi: 10.1007/978-3030-00776-8_62

Stockwell, R. G. (1996). Localization of the complex spectrum: the s transform. IEEE Transactions on Signal Processing, 44(4), 993. doi: 10.1109/78.492555

Stockwell, R. G. (2007). A basis for efficient representation of the S-transform. Digital Signal Processing: A Review Journal, 17(1), 371-393. doi: 10.1016/j.dsp.2006.04.006

Wang, X., Liu, A., Zhang, Y., \& Xue, F. (2019). Underwater acoustic target recognition: A combination of multidimensional fusion features and modified deep neural network. Remote Sensing, 11(16). doi: 10.3390/rs11161888

Yaman, O., \& Tuncer, T. (2021). Ensemble NASNet Deep Feature Generator Based Underwater Acoustic Classification Model. Veri Bilimi, 4(2), 33-39.

Yaman, O., Tuncer, T., \& Tasar, B. (2021). DES-Pat: A novel DES pattern-based propeller recognition method using underwater acoustical sounds. Applied Acoustics, 175, 107859. doi: 10.1016/j.apacoust.2020.107859 